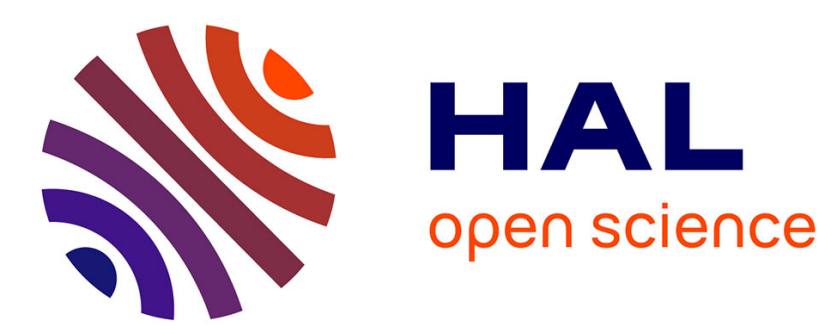

\title{
Application du système d'acquisition de données Plurimat 20 en physique
}

J.F. Sellier, G. Guillaume, J.P. Weymann

\section{To cite this version:}

J.F. Sellier, G. Guillaume, J.P. Weymann. Application du système d'acquisition de données Plurimat 20 en physique. Revue de Physique Appliquée, 1977, 12 (2), pp.447-450. 10.1051/rphysap:01977001202044700 . jpa-00244195

\section{HAL Id: jpa-00244195 https://hal.science/jpa-00244195}

Submitted on 1 Jan 1977

HAL is a multi-disciplinary open access archive for the deposit and dissemination of scientific research documents, whether they are published or not. The documents may come from teaching and research institutions in France or abroad, or from public or private research centers.
L'archive ouverte pluridisciplinaire HAL, est destinée au dépôt et à la diffusion de documents scientifiques de niveau recherche, publiés ou non, émanant des établissements d'enseignement et de recherche français ou étrangers, des laboratoires publics ou privés. 
Classification

Physics Abstracts

0.670

\title{
APPLICATION DU SYSTÈME D'ACQUISITION De DONNÉES PLURIMAT 20 EN PHYSIQUe
}

\author{
J. F. SELlier $(*)$, G. GUILlAUME et J. P. WEYMANN \\ Centre de Recherches Nucléaires et Université Louis Pasteur, Strasbourg, France
}

(Reçu le 24 septembre 1976, accepté le 27 octobre 1976)

\begin{abstract}
Résumé. - Deux applications du système d'acquisition de données Plurimat 20 sont présentées. L'une concerne un appareil destiné à l'enregistrement automatique sur minicassette de 48 paramètres indépendants, quasi simultanément, provenant de mesures de grandeurs physiques (telles que des températures) codées en fréquence. L'échantillonnage des paramètres et le couplage de la minicassette au Plurimat 20 sont décrits. L'autre application a trait à l'acquisition d'événements corrélés en Physique Nucléaire ; une expérience de corrélation angulaire $\alpha-\gamma$, par la réaction ${ }^{18} \mathrm{O}(\mathrm{t}, \alpha \gamma)^{17} \mathrm{~N}$, est décrite dans sa partie technique.
\end{abstract}

\begin{abstract}
Two applications of the Plurimat 20 data-acquisition system are presented. The first concerns the apparatus used to automatically and quasi-simultaneously record 48 independent physical parameters (such as temperatures) on a minicassette. The sampling procedure and the coupling of the cassette to the Plurimat 20 are described. The second application treats the acquisition of correlated nuclear physics events and is exemplified by an $\alpha-\gamma$ angular correlation experiment using the ${ }^{18} \mathrm{O}(\mathrm{t}, \alpha \gamma){ }^{17} \mathrm{~N}$ reaction.
\end{abstract}

1. Le système Plurimat 20. - Nous présentons ici brièvement et pour la clarté de l'exposé qui suit, le système d'acquisition Plurimat $20\left(^{1}\right)$ utilisé dans notre laboratoire. Ce système comprend un miniordinateur Multi 20 de $32 \mathrm{~K}$ octets de mémoire, une visualisation biparamétrique, un dérouleur de bande magnétique 9 pistes 800 BPI Type AMPEX-TMZ compatible IBM, deux convertisseurs analogiquesnumériques CT 103 Intertechnique et une télétype ASR 33. La liaison entre le mini-ordinateur et les périphériques est effectuée par l'intermédiaire d'un ensemble de coupleurs soit sur la liaison standard parallèle qui est constituée du bus d'entrée-sortie, soit sur la mémoire par canal d'accès direct mémoire. Un coupleur complémentaire M 1604, 32 entrées et sorties numériques, résout la plupart des connexions d'éléments périphériques non conventionnels. Ces 32 lignes sont réparties en 4 groupes de 8 lignes. Chaque groupe constitue un canal adressable grâce aux codes de fonction des instructions d'entrée-sortie. Les transferts de données s'effectuent en mode programmé sur le bus d'entrée-sortie parallèle. Chacun des 4 canaux dispose d'une ligne d'interruption ordonnée par le périphé-

$\left(^{*}\right)$ Adresse permanente : Ecole Nationale Supérieure des Arts et Industries, Strasbourg, France.

(1) Système commercialisé par la Société Intertechnique, 78370 Plaisir, France. rique. Un $O U$ logique réunit les 4 lignes d'interruption en un niveau unique d'interruption externe commandé par un sous-programme d'interruption.

Des dispositifs électroniques originaux et complémentaires, tels que enregistrement de données sur minicassette du commerce et relecture de ces cassettes sur le mini-ordinateur Multi 20, sont décrits en détail ci-après.

\section{Applications du système Plurimat 20 en physique.} - 2.1 CODAGE EN FRÉQUENCE D'INFORMATIONS ISSUES DE CAPTEURS DE TEMPÉRATURE ET STOCKAGE SUR MINICASSETTE DU COMMERCE. - 2.1.1 Introduction. La réalisation de circuits électroniques annexes au mini-ordinateur et l'utilisation de ce dernier est en relation avec le problème physique suivant : comment déterminer les coefficients d'échange thermique d'une enceinte de grand volume (par exemple un immeuble) ? Il est pour cela nécessaire de connaître les variations des caractéristiques thermiques de cette enceinte avec les conditions extérieures, en effectuant des mesures de température en de nombreux endroits de l'enceinte. Par suite du grand nombre de mesures à faire et de leur durée (plusieurs jours ou même plusieurs semaines) il est nécessaire de prévoir un système d'acquisition de données à grande capacité mémoire. Pour éviter l'immobilisation permanente du système d'acquisition de données, nous avons stocké les informations, après 
codage en fréquence, sur une minicassette. Nous avons réalisé une interface branchée sur le coupleur M 1604, qui effectue le vidage des événements enregistrés sur la minicassette dans la mémoire du mini-ordinateur Multi 20. L'enregistrement sur bande magnétique 9 pistes 800 BPI permet alors un traitement mathématique élaboré des événements sur des ordinateurs de plus grosse taille (UNIVAC 1100, CII du CRN, par exemple).

2.1.2 Caractéristiques des paramètres et de l'échantillonnage. - Les températures sont des grandeurs physiques ramenées à une grandeur électrique analogique ou digitale à l'aide de transducteurs appropriés. Nous avons entrepris de pouvoir suivre l'évolution de 48 paramètres indépendants, quasi simultanément et de les enregistrer. Un commutateur analogique et un convertisseur analogique-numérique permettent de coder successivement tous les paramètres. L'enregistrement de la valeur mesurée s'effectue alors immédiatement à la fin du temps de mesure. La rapidité de l'échantillonnage dépend surtout du convertisseur analogique-numérique utilisé. Dans notre cas, le temps de mesure est de $100 \mathrm{~ms}$ et le temps d'enregistrement a été fixé à la même valeur pour permettre l'enregistrement de tous les bits caractérisant un paramètre. La scrutation de tous les paramètres s'effectue régulièrement et le temps entre chaque scrutation peut être présélectionné à une valeur comprise entre 1 et 90 minutes. L'enregistreur ne fonctionne pas continuellement mais est mis en route à chaque scrutation et arrêté après le $48^{\mathrm{e}}$ paramètre. Le diagramme en temps de l'enregistrement est explicité par le schéma de la figure 1.

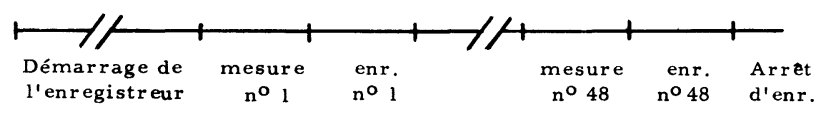

FIG. 1. - Diagramme en temps d'un enregistrement complet.

\subsubsection{Caractéristiques de l'enregistrement. -} Comme le temps d'enregistrement d'un paramètre est relativement long, on a choisi pour représenter les nombres un codage en fréquence. Aux valeurs 1 et 0 de l'information digitale correspond respectivement les fréquences $f_{1}$ et $f_{0}$. Un digit de fréquence $f_{2}$, intercalé entre les digits $f_{1}$ et $f_{0}$, permet le synchronisme de la bande magnétique à la lecture. Ce nombre digital représentant la valeur d'un paramètre est codé sur 25 bits appelés bits informations. Entre chaque bit information est intercalé un bit horloge pour la synchronisation. Au total, il y a 50 bits par nombre. Le temps d'enregistrement d'un nombre est de $100 \mathrm{~ms}$, la durée d'un bit sera donc de $2 \mathrm{~ms}$. Celle-ci étant fixée, on peut choisir les fréquences $f_{0}, f_{1}, f_{2}$ qui ont pour valeur respectivement $0,4000 \mathrm{~Hz}, 6000 \mathrm{~Hz}$. La valeur 0 correspond à l'absence de signal. La figure 2 représente le diagramme d'enregistrement d'un nombre.

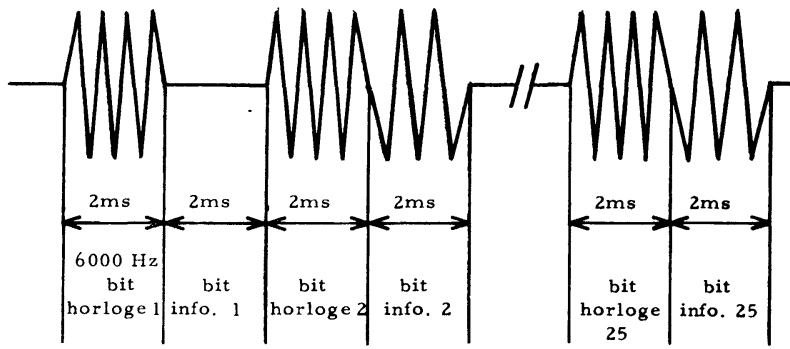

Fig. 2. - Diagramme de l'enregistrement d'un nombre.

Après l'enregistrement d'un nombre, il y a donc un espace de $100 \mathrm{~ms}$ correspondant à une mesure. On place dans cet intervalle de temps une information supplémentaire de fréquence $f_{3}$ indiquant la fin de l'enregistrement du nombre comme le montre le diagramme de la figure 3.

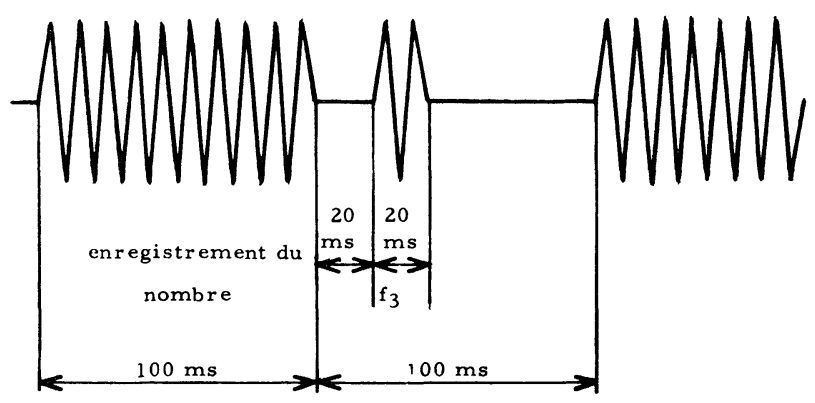

FIG. 3. - Diagramme en temps de la fin d'un enregistrement.

2.1.4 Lecture de la bande. - La sélection de chaque fréquence s'effectue à l'aide de filtres. Chaque filtre est suivi d'un circuit de mise en forme composé d'un comparateur et d'un monostable (Fig. 4).

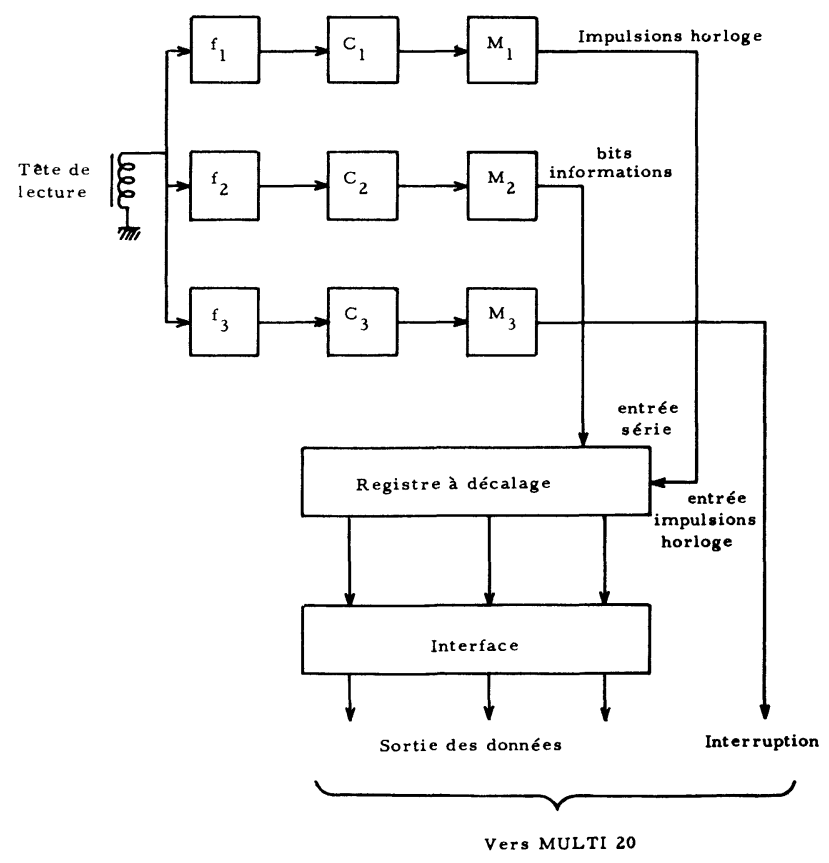

Fig. 4. - Schéma de principe du lecteur ; $\mathrm{f}=$ filtre, $\mathrm{C}=$ comparateur, $\mathrm{M}=$ monostable. 
Un registre à décalage transforme l'information série en une information parallèle. La sortie parallèle du registre est reliée à l'entrée de la carte coupleur M 1604 du mini-ordinateur Multi-20. La sortie fin de mot envoie une impulsion sur la ligne d'interruption du mini-ordinateur. Ce dernier traite alors un sousprogramme correspondant à cette interruption. Le programme en langage machine comporte donc deux phases : l'attente d'interruption et le sous-programme d'interruption entrée du nombre. Le programme est disponible sous forme de ruban perforé. Le sousprogramme d'interruption effectue le cadrage du nombre et le rangement en mémoire. Il est possible de prévoir un sous-programme de traitement plus complet tel que linéarisation et calibration des nombres. Toutefois, ce programme ne peut durer plus de $200 \mathrm{~ms}$, puisque au bout de ce temps un nouveau nombre doit pouvoir entrer en mémoire.

2.1.5 Conclusion. - Bien que notre réalisation soit caractérisée par une faible rapidité d'acquisition (5 paramètres par seconde), elle a l'avantage d'un faible coût de revient par suite de la simplicité de la conception des circuits électroniques et d'être insensible aux parasites ambiants. Ultérieurement, nous augmenterons la rapidité et la fiabilité de l'enregistrement par l'utilisation de bande magnétique à deux pistes, l'une pour les informations et l'autre pour les bits de synchronisation.

2.2 Corrélations ANGUlaires DANS LE NOYAU ${ }^{17} \mathrm{~N}$. - La détermination de moments angulaires totaux (spins) des états excités d'un noyau, ainsi que de mélanges multipolaires des transitions électromagnétiques, requiert le plus souvent la mesure de fonctions de corrélation angulaire entre deux radiations successives. Dans le cas du noyau ${ }^{17} \mathrm{~N}$, que nous avons étudié par la réaction ${ }^{18} \mathrm{O}(\mathrm{t}, \alpha \gamma){ }^{17} \mathrm{~N}$ à $E_{\mathrm{t}}=3.5 \mathrm{MeV}$ [1], les corrélations angulaires ont été mesurées dans une géométrie particulière dite méthode $I I$ de Litherland et Ferguson [2] : seules les particules $\alpha$ émises le long de l'axe du faisceau des tritons incidents étaient détectées, en coïncidence avec les rayonnements $\gamma$ produits par la réaction. Dans cette symétrie axiale, seuls les sous-états magnétiques $+1 / 2$ et $-1 / 2$ sont peuplés, à égalité ; on réalise ainsi un alignement optimum des noyaux ${ }^{17} \mathrm{~N}^{*}$. En réalité, le détecteur de particules (annulaire, en silicium) que nous avions placé à $180^{\circ}$, a des dimensions finies, avec des angles de détection compris entre $164^{\circ}$ et $175^{\circ}$, ce qui a pour effet de peupler aussi, et pour moins de $10 \%$ les sous-états magnétiques $+3 / 2$ et $-3 / 2$. La détection des rayonnements $\gamma$ était assurée par un cristal $\mathrm{Ge}(\mathrm{Li})$ de $120 \mathrm{~cm}^{3}$ de volume et de $4 \mathrm{keV}$ de résolution en énergie à $E_{\gamma}=1,33 \mathrm{MeV}$ (sous faisceau), placé à $9 \mathrm{~cm}$ de la cible, et tour à tour positionné à $\theta_{\gamma}=0^{\circ}, 30^{\circ}, 45^{\circ}, 55^{\circ}$, $60^{\circ}$ et $90^{\circ}$. Tous les détecteurs utilisés ont été construits dans notre laboratoire. La cible était constituée d'une feuille autoportée de $50 \mu \mathrm{g} / \mathrm{cm}^{2}$ d'oxyde de titane; elle a été préalablement fabriquée, sous enceinte d'argon, par électrolyse d'une feuille de titane dans un bain $\mathrm{D}_{2}{ }^{18} \mathrm{O}$ dont l'enrichissement en ${ }^{18} \mathrm{O}$ était de $98 \%$. $\mathrm{La}$ réaction ${ }^{18} \mathrm{O}(\mathrm{t}, \alpha){ }^{17} \mathrm{~N}$ est caractérisée par un rendement faible [3], mais est certainement la seule utilisable pour lever toutes les ambiguïtés laissées par les études antérieures sur ${ }^{17} \mathrm{~N}$. Les réactions compétitives ou parasites, nombreuses et prolifiques $\left[{ }^{18} \mathrm{O}(\mathrm{t}, \mathrm{p}){ }^{20} \mathrm{O}\right.$, ${ }^{16} \mathrm{O}(\mathrm{t}, \mathrm{p}){ }^{18} \mathrm{O},{ }^{12} \mathrm{C}(\mathrm{t}, \mathrm{p}){ }^{14} \mathrm{C}$ notamment $]$, ainsi que le bruit de rayonnement ambiant occasionné par l'accélérateur et le faisceau de tritons, masquent les phénomènes à étudier. De façon à remédier à ceci, nous avons utilisé d'une part, un détecteur de particules mince (d'épaisseur $34 \mu \mathrm{m}$ dans une première série de mesures, puis un autre de $25 \mu \mathrm{m}$ dans la suite) permettant l'obtention d'un spectre de particules $\alpha$ débarrassé des groupes de protons sur sa plus grande partie et, d'autre part, le système Plurimat 20 permettant l'acquisition de tous les événements en mode biparamétrique sur des temps de mesure longs. Soulignons que ce logiciel programmable évitait aussi l'emploi de fenêtres analogiques dont la multiplication n'aurait eu d'autres fins que de diminuer la fiabilité de l'expérience et de donner des informations partielles et achevées qui ne pourraient être ni améliorées, ni corrigées qu'aux dépens de nouvelles semaines de mesure à l'accélérateur.

Les circuits électroniques utilisés (Fig. 5) sont du type rapide-lent avec un temps de résolution de $30 \mathrm{~ns}$

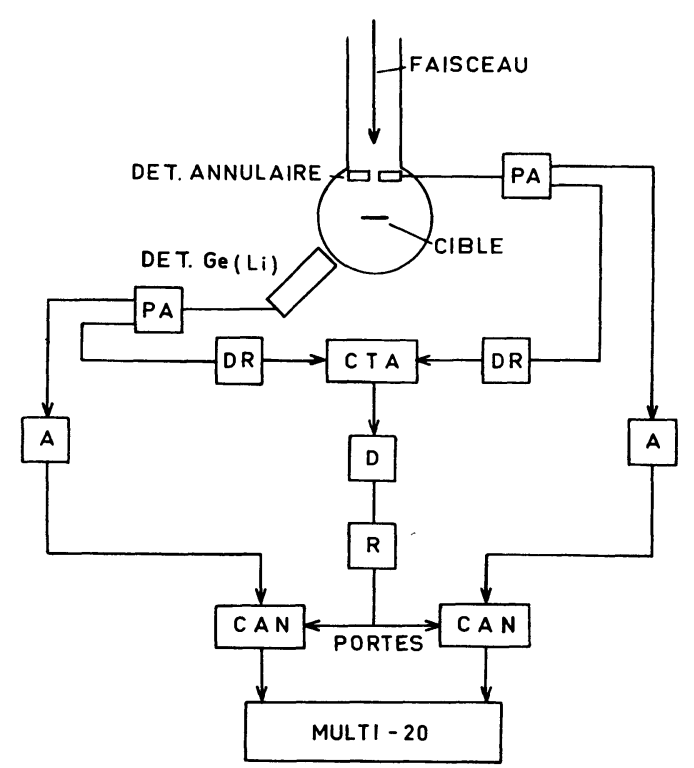

Fig. 5. - Bloc diagramme de l'unité de coïncidence « rapidelent ». $\mathbf{P A}=$ préamplificateur ; $\mathbf{A}=$ amplificateur linéaire ; $\mathrm{DR}=$ discriminateur à fraction constante $; \mathrm{CTA}=$ convertisseur temps amplitude; $\mathrm{D}=$ discriminateur différentiel ; $\mathrm{R}=$ ligne à retard ; CAN = convertisseur analogique-numérique.

(mesuré à la base du pic temps donné par le CTA et sans sélection d'énergies sur les deux voies) et un rapport vrais/fortuites meilleur que 20 . Le signal de coïncidence rapide, chaque fois qu'il se produit, ouvre 
la porte des deux convertisseurs analogiques CT 103 laissant le passage à deux impulsions énergies venant des deux détecteurs. Au niveau de l'ordinateur Multi-20 des couples $(X, Y)=($ énergie gamma, énergie particules) sont ainsi générés et codés sur deux fois deux octets. La configuration maximum que nous avons adoptée était de $2048 \times 512$ canaux. Une mémoire tampon dans l'ordinateur retient ces couples en simultanéité avec l'acquisition et est vidée, lorsqu'elle est saturée, sur une bande magnétique, pendant que, sans temps mort, une autre mémoire s'active. Des blocs de 960 octets sont ainsi constitués sur la bande magnétique, les deux premiers octets étant réservés à la date d'écriture du bloc [4].

L'expérience a duré environ trois semaines. Le taux de coïncidences était de l'ordre de un à deux coups par seconde pour un faisceau de tritons d'intensité 20 à $80 \mathrm{nA}$ suivant l'angle $\theta_{\gamma}$ choisi; ces conditions correspondaient en fait à des temps morts d'environ $60 \%$ au niveau des convertisseurs CT 103 et pour les spectres directs.

Les événements biparamétriques stockés sur bandes magnétiques ont été ensuite triés et ordonnés en plaçant, par programme, des fenêtres numériques sur les voies d'intérêt $X$ ou $Y$. La reconstitution des spectres en coïncidence, soit par feuilletage, soit par projection, a été réalisée à cause d'une infrastructure en programmes divers très élaborée, sur un ordinateur IBM 1130 et dont la structure en mot de 16 bits permet une lecture aisée des événements. La figure 6 montre

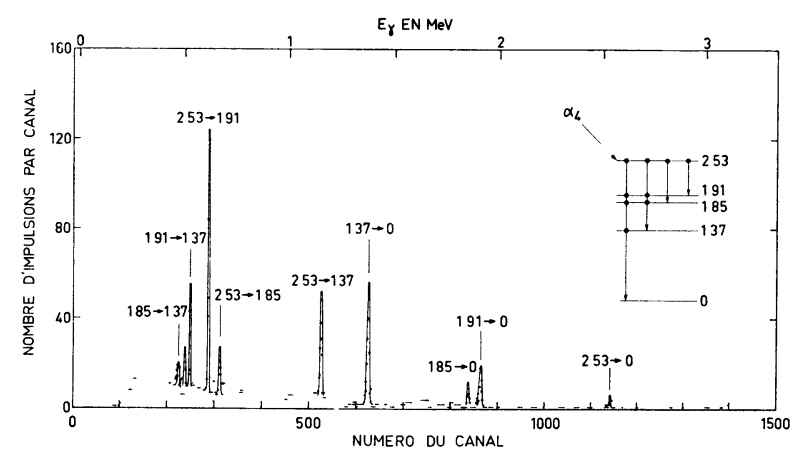

Fig. 6. - Spectre de rayonnements $\gamma$ obtenu à $\theta_{\gamma}=90^{\circ}$ en coïncidence avec le groupe de particules $\alpha$ alimentant le niveau de 2,53 MeV. Ce spectre, construit à partir des événements bruts, représente environ le tiers de la statistique totale. Le schéma de désexcitation du niveau de $2,53 \mathrm{MeV}$ est présenté en encart.

un spectre de rayonnements $\gamma$ en coïncidence, reconstruit en plaçant une fenêtre en $Y$ sur les groupes de particules $\alpha_{4}$ alimentant le niveau de $2,53 \mathrm{MeV}$ de ${ }^{17} \mathrm{~N}$. Les intensités des pics de pleine énergie ont été extraits pour chaque angle $\theta_{\gamma}$ et normalisées d'un angle à un autre à l'aide des spectres de particules directs. Pour l'obtention des informations de nature spectroscopique, nous avons analysé les corrélations angulaires suivant le formalisme développé par Poletti et Warburton [5] : les corrélations angulaires expérimentales sont lissées par des fonctions de corrélations angulaires théoriques contenant les quantités à obtenir (spins, mélanges multipolaires, intensités) et par une méthode de moindres carrés linéaires.

Un travail assez long de dépouillement des résultats bruts, qui a consisté à relire les bandes magnétiques de nombreuses fois à l'ordinateur, nous a permis de déterminer, sans ambiguïté, les propriétés électromagnétiques des six premiers états excités de ${ }^{17} \mathrm{~N}$ [1]. Ces propriétés, telles que énergies des états, rapports d'embranchement des transitions $\gamma$, mélanges multipolaires et valeurs de spins, découlent de notre étude sans référence aux résultats fragmentaires connus par ailleurs. Un intérêt évident d'un système mégacanaux, avec stockage de tous les événements sur bande magnétique, réside aussi dans le fait que des résultats antérieurement bien connus et qui concernent d'autres noyaux, peuvent être recherchés et servir de tests de confiance. Ainsi les énergies des états de ${ }^{17} \mathrm{~N}$ sont établies, à $\theta_{\gamma}=90^{\circ}$, par référence avec ceux de ${ }^{11} \mathrm{~B}$ et ${ }^{18} \mathrm{O}$, en particulier, atteints par les réactions ${ }^{12} \mathrm{C}(\mathrm{t}, \alpha \gamma){ }^{11} \mathrm{~B}$ et ${ }^{16} \mathrm{O}(\mathrm{t}, \mathrm{p} \gamma){ }^{18} \mathrm{O}$; les spins de quelques niveaux très excités de ${ }^{20} \mathrm{O}$, atteints par ${ }^{18} \mathrm{O}(\mathrm{t}, \mathrm{p}){ }^{20} \mathrm{O}$, sont aussi retrouvés en traitant les corrélations angulaires $(p, \gamma)$ correspondantes.

3. Conclusion. - Nous avons décrit dans cet article deux applications du système d'acquisition Plurimat 20. L'une concernait l'enregistrement, effectué automatiquement et quasi simultanément, de 48 paramètres indépendants sur minicassette et le couplage de cette dernière au système. Il est bien entendu que les 48 paramètres (ou plus) peuvent provenir d'autres grandeurs physiques que des températures, en faisant suivre les appareils de mesures de transducteurs ou de convertisseurs appropriés. La seconde application a été faite à la Physique Nucléaire où le système Plurimat 20 a montré sa nécessité par rapport aux systèmes classiques modulaires, sa facilité d'utilisation, son utilité et sa fiabilité à l'acquisition de grandeurs corrélées, telles que celles provenant d'une expérience de corrélations angulaires particule-gamma.

Remerciements. - Un des auteurs (JFS) est reconnaissant au Professeur R. Armbruster de l'avoir accueilli dans son laboratoire au C. R. N.

\section{Bibliographie}

[1] Guillaume, G., Rastegar, B., Fintz, P. et Gallmann, A., Nucl. Phys., sous presse.

[2] Litherland, A. E. and Ferguson, A. J., Can. J. Phys. 39 (1961) 788.
[3] Jarmie, N. and Silbert, M. G., Phys. Rev. 120 (1960) 914. [4] Programme PN05A/012D standart, fourni par le constructeur (Société Intertechnique).

[5] Poletti, A. R. and Warburton, E. K., Phys. Rev. 137 (1965) B 595. 